\title{
Caffeic acid, morin hydrate and quercetin partially attenuate sulfur mustard-induced cell death by inhibiting the lipoxygenase pathway
}

\author{
SHIN KIM ${ }^{1,2}$, KWANG-JOON JEONG ${ }^{2,3}$, SUNG KWEON CHO ${ }^{2}$, JOO-WON PARK ${ }^{4}$ and WOO-JAE PARK ${ }^{2,5}$ \\ ${ }^{1}$ Department of Immunology, School of Medicine, Keimyung University, Daegu 406-799; ${ }^{2}$ Department of Research, \\ The Armed Forces Medical Research Institute, Daejeon 305-878; ${ }^{3}$ Department of Microbiology, Chonnam National University \\ Medical School, Gwangju 501-746; ${ }^{4}$ Department of Biochemistry, School of Medicine, Ewha Womans University, \\ Seoul 158-710; ${ }^{5}$ Department of Biochemistry, School of Medicine, Gachon University, Incheon 406-799, Republic of Korea
}

Received September 4, 2015; Accepted September 5, 2016

DOI: $10.3892 / \mathrm{mmr} .2016 .5766$

\begin{abstract}
Sulfur mustard (SM) is an alkylating agent, which has been used as in chemical warfare in a number of conflicts. As the generation of reactive oxygen species (ROS), and adducts in DNA and proteins have been suggested as the mechanism underlying SM-induced cytotoxicity, the present study screened several antioxidant candidates, including tannic acid, deferoxamine mesylate, trolox, vitamin $\mathrm{C}$, ellagic acid and caffeic acid (CA) to assess their potential as therapeutic agents for SM-induced cell death. Among several antioxidants, CA partially alleviated SM-induced cell death in a dose-dependent manner. Although CA treatment decreased the phosphorylation of p38 mitogen-activated protein (MAP) kinase and p53, p38 MAP kinase inhibition by SB203580 did not affect SM-induced cell death. As CA has also been reported as a 15-lipoxygenase (15-LOX) inhibitor, the role of 15-LOX in SM-induced cytotoxicity was also examined. Similar to the results observed with CA, treatment with PD146176, a specific 15-LOX inhibitor, decreased SM-induced
\end{abstract}

Correspondence to: Dr Woo-Jae Park, Department of Biochemistry, School of Medicine, Gachon University, Yeonsu-3-Dong, Yeonsugu, Incheon 406-799, Republic of Korea

E-mail: ooze@gachon.ac.kr

Dr Joo-Won Park, Department of Biochemistry, School of Medicine, Ewha Womans University, 911-1 Mok-Dong, Yang Cheon-Gu, Seoul 158-710, Republic of Korea

E-mail: joowon.park@ewha.ac.kr

Abbreviations: SM, sulfur mustard; CA, caffeic acid; COX2, cyclooxygenase 2; HETE, hydroxyeicosatetraenoic acid; IL-1 $\beta$, interleukin-1 $\beta$; iNOS, inducible nitric oxide synthase; LOX, lipoxygenase; ROS, reactive oxygen species; TNF- $\alpha$, tumor necrosis factor- $\alpha$; RT-CES, real-time cell electronic sensing

Key words: caffeic acid, sulfur mustard, toxicity, lipoxygenase, morin hydrate, quercetin cytotoxicity, accompanied by decreases in the production of tumor necrosis factor- $\alpha$ and 15-hydroxyeicosatetraenoic acid. Furthermore, the present study investigated the protective effects of two natural 15-LOX inhibitors, morin hydrate and quercetin, in SM-induced cytotoxicity. As expected, these inhibitors had similar protective effects against SM-induced cytotoxicity. These antioxidants also reduced the generation of ROS and nitrate/nitrite. Therefore, the results of the present study indicated that the natural products, CA, quercetin and morin hydrate, offer potential as adjuvant therapeutic agents for SM-induced toxicity, not only by reducing inflammation mediated by the p38 and LOX signaling pathways, but also by decreasing the generation of ROS and nitrate/nitrite.

\section{Introduction}

Sulfur mustard (SM; 2,2'-dichlorodiethyl sulfide), commonly known as mustard gas, is a potent bifunctional alkylating agent (1), and was used as a chemical weapon during World War I (1914-1918). Although more toxic chemical warfare agents are currently available, mustard gas has remained the chemical weapon of choice in modern warfare, as evidenced by its use during the Iran-Iraq war between 1980 and 1988 (2). SM is a reactive chemical causing skin, ocular and pulmonary damage (1). Among these, the primary target organ of SM is the skin, which exhibits erythema, hyperpigmentation, inflammation, blistering and severe necrosis (1). As no effective treatment for the vesicant-inducing properties of SM has been established (3), the development of novel therapeutic agents for SM-induced toxic symptoms is clinically urgent considering the possibility of its use during future conflict.

Various molecules have been suggested to be involved in the mechanism of SM-induced toxicity. Firstly, SM causes DNA damage, which activates the poly (ADP-ribose) polymerase (PARP) nuclear protein $(1,4)$. The increased activation of PARP depletes NAD ${ }^{+}$and intracellular ATP levels, leading to necrotic cell death. SM-induced DNA damage can also be caused by its DNA adducts, which are formed predominantly at the N7-position of guanine and the N1-/N3-position of adenine (1). These DNA adducts eventually cause cell cycle 
arrest, leading to cell death. Secondly, SM induces apoptotic cell death by increasing the expression levels of Fas receptor, Fas ligands, tumor necrosis factor (TNF) receptor ligands and TNF- $\alpha$. SM also activates caspase- 8 and its downstream caspases, including caspase-3, -6 and -7 (1). Thirdly, endoplasmic reticulum stress with changes in calcium homeostasis has also been reported to be induced by SM through the modulation of calcium-calmodulin signaling (1,5). SM can also alter signaling pathways, including p38 mitogen-ativated protein (MAP) kinase and matrix metalloproteases (MMPs). The SM-induced activation of p38 MAP kinase is involved in cytokine release (6) and MMPs are important in SM-induced skin blistering (1). Furthermore, SM activates nuclear factor (NF) $-\kappa B$ signaling (7), and increases the generation of reactive oxygen species (ROS) and nitric oxide, which depletes glutathione (8). Therefore, antioxidants have been used as therapeutic agents for SM-induced toxicity (9-11). However, the therapeutic efficacy of antioxidants is not clinically satisfactory and current treatments are only supportive.

Historically, therapeutic agents were predominantly obtained from various plants, and natural selection and competition among species led to the synthesis of secondary metabolites with marked biological activities (12). Caffeic acid (CA), an active component of propolis, which has anti-inflammatory and anticarcinogenic properties (13), and inhibits 15-lipoxygenase (LOX) (14). Several other natural 15-LOX inhibitors, including quercetin (15), a flavonoid found in a number of vegetables and fruits, and morin hydrate, a yellow substance found in oranges and guava (16), have been reported previously.

LOX is an enzyme catalyzing the deoxygenation of polyunsaturated fatty acids in lipids containing a cis, cis-1,4-pentadiene structure, which generates the fatty acid, hydroperoxide (17). LOX reactions can modulate signaling pathways via the generation of leukotrienes or 12-hydroxyeicosatetraenoic acid (12-HETE), but can also induce structural or metabolic alterations inside the cell (17). In the present study, it was demonstrated that CA, quercetin and morin hydrate not only reduced SM-induced inflammatory cytokines via 15 -LOX inhibition, but also reduced SM-induced oxidative stress and the generation of nitrate/nitrite.

\section{Materials and methods}

Materials. CA, tannic acid (TA), deferoxamine mesylate, trolox, vitamin $\mathrm{C}$, ellagic acid, $\mathrm{N}$-acetyl-L-cysteine, quercetin, morin hydrate, SB203580, PD146176 and 2',7'-dichlorofluorescein diacetate (DCF-DA) were purchased from Sigma-Aldrich; Thermo Fisher Scientific, Inc. (Waltham, MA, USA). Antibodies detecting Thr183/Thr185-phosphorylated-c-jun N-terminal kinases (JNKs; cat. no. 9255), JNKs (cat. no. 9252), Thr180/Tyr182-phosphorylated-p38 (cat. no. 4511), p38, Ser1 5-phosphorylated-p53 (cat. no. 9284), p53 (cat. no. 2524) and cyclooxygenase 2 (COX2; cat. no. 12282), all purchased from Cell Signaling Biotechnology (Beverly, MA, USA), and inducible nitric oxide synthase (iNOS; cat. no. ab3523; Abcam, Cambridge, MA, USA). 12-LOX (cat. no. sc-32939) and 15-LOX (cat. no. sc-67143) antibodies were obtained from Santa Cruz Biotechnology, Inc. (Santa Cruz, CA, USA). Glyceraldehyde-3-phosphate dehydrogenase (GAPDH; cat. no. MAB374) antibody was purchased from EMD
Millipore (Billerica, MA, USA). Anti-mouse-horseradish peroxidase (HRP; cat. no. 115-036-003) and anti-rabbit-HRP (cat. no. 111-035-003) antibodies were purchased from Jackson Laboratory (Bar Harbor, ME, USA). The SM was provided from the Agency for Defense Development (Daejeon, Korea).

Cell culture. Normal human epithelial keratinocytes (NHEKs) were purchased from ZenBio, Inc. (Research Triangle Park, NC, USA). The NHEKs were grown in KM-3 growth medium (ZenBio, Inc.) in $5 \% \mathrm{CO}_{2}$ at $37^{\circ} \mathrm{C}$. All experiments were performed with NHEKs at passages 3-5.

Measurements of TNF- $\alpha$, interleukin-1 $\beta$ (IL-1 $\beta)$ and 15-hydroxyeicosatetraenoic acid (15-HETE). The NHEKs at $80 \%$ confluence were treated with $200 \mu \mathrm{M}$ of SM with or without antioxidants. After $18 \mathrm{~h}$, the levels of TNF- $\alpha$, IL- $1 \beta$ and 15-HETE in the culture medium were measured using TNF- $\alpha$ and IL-1 $\beta$ Human ELISA kits (AbFrontier, Seoul, Korea) and a 15-HETE ELISA kit (Abcam, Cambridge, MA, USA) according to the manufacturer's protocols.

Western blot analysis. The NHEKs were lysed using RIPA buffer containing 50 mM Tris- $\mathrm{Cl}$ (pH 7.5), $150 \mathrm{mM}$ $\mathrm{NaCl}, 1 \%$ Nonidet $\mathrm{P}-40,0.5 \%$ sodium deoxycholate, $0.1 \%$ SDS, protease inhibitors and phosphatase inhibitors (Sigma-Aldrich; Thermo Fisher Scientific, Inc.) and protein levels were examined using Protein Assay Dye reagent (Bio-Rad Laboratories, Inc., Hercules, CA, USA). The proteins $(50 \mu \mathrm{g})$ were separated by SDS-PAGE on $8-15 \%$ SDS polyacrylamide gels and transferred onto PVDF membranes (Bio-Rad Laboratories, Inc.). The membranes were blocked with the $5 \%$ skim milk for $1 \mathrm{~h}$ and incubated with primary antibodies overnight at $4^{\circ} \mathrm{C}$. The membranes were then incubated with secondary antibodies for $1 \mathrm{~h}$ at room temperature. The protein bands were detected on X-ray film using ECL Western Blotting Detection reagents (GE Healthcare Life Sciences, Little Chalfont, UK).

3-(4,5-dimethylthiazol-2-yl)-2,5-diphenyltetrazolium bromide (MTT) cell viability assay. Cell viability was determined using an MTT assay. Briefly, 5x10 ${ }^{4}$ NHEKs were grown overnight in 96-well plates, and were treated with 0-800 $\mu \mathrm{M} \mathrm{SM}$ for $24 \mathrm{~h}$ with or without antioxidants (10 $\mu \mathrm{M}$ TA, $250 \mu \mathrm{M}$ DFM, $250 \mu \mathrm{M}$ Trolox, $100 \mathrm{mM}$ vitamin $\mathrm{C}, 250 \mu \mathrm{M}$ EA, $250 \mu \mathrm{M} \mathrm{CA}$ and $10 \mathrm{mM} \mathrm{NAC).} \mathrm{Following} \mathrm{treatment,} \mathrm{MTT} \mathrm{solution} \mathrm{was}$ added $(0.5 \mathrm{mg} / \mathrm{ml}$ final concentration), and incubated for $4 \mathrm{~h}$ at $37^{\circ} \mathrm{C}$ with $5 \% \mathrm{CO}_{2}$. The supernatant was discarded and $200 \mu \mathrm{l}$ dimethyl sulfoxide was added. The production of solubilized purple formazan crystals was quantified by exposure to a wavelength of $540 \mathrm{~nm}$.

Real-time cell electronic sensing (RT-CES). The NHEKs $\left(5 \times 10^{4}\right)$ were incubated in 16 plastic wells with $16 \mathrm{X}$ microelectronic sensor devices. At $5 \mathrm{~h}$ post-cell attachment, $200 \mu \mathrm{M} \mathrm{SM}$ with or without $250 \mu \mathrm{M} \mathrm{CA}, 100 \mu \mathrm{M}$ quercetin or $250 \mu \mathrm{M}$ morin hydrate, was added. Data was collected every 3 min using an RT-CES system (ACEA Biosciences, San Diego, CA, USA).

ROS measurement and nitrate/nitrite assay. The NHEKs treated with $200 \mu \mathrm{M}$ SM with or without antioxidants 
A

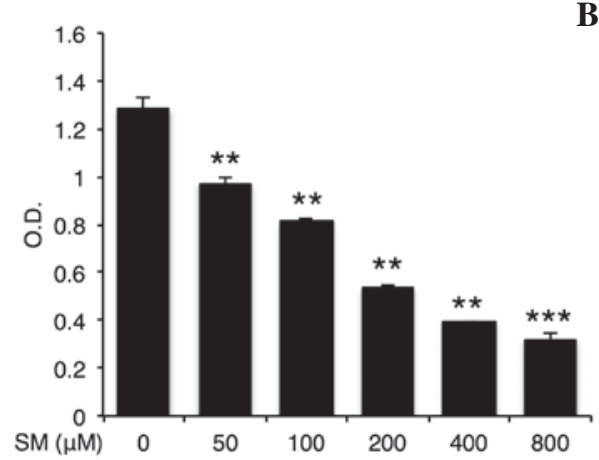

B

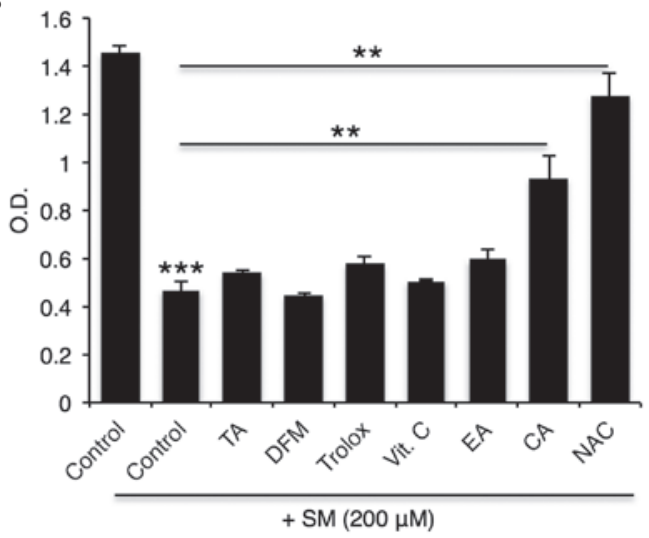

C

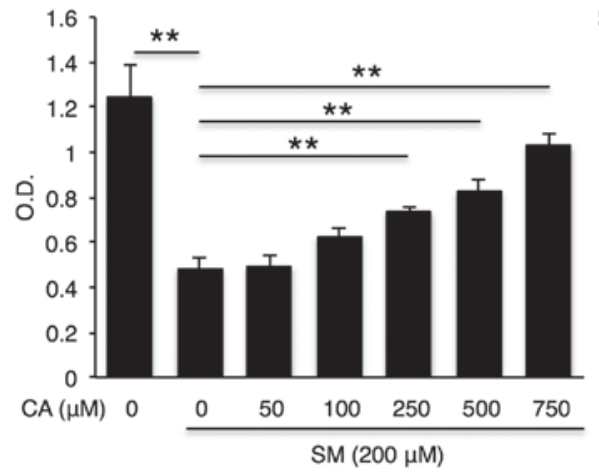

D $\quad \mathrm{CA}(\mu \mathrm{M}) \quad 0 \quad 125 \quad 250 \quad 0 \quad 125 \quad 250$

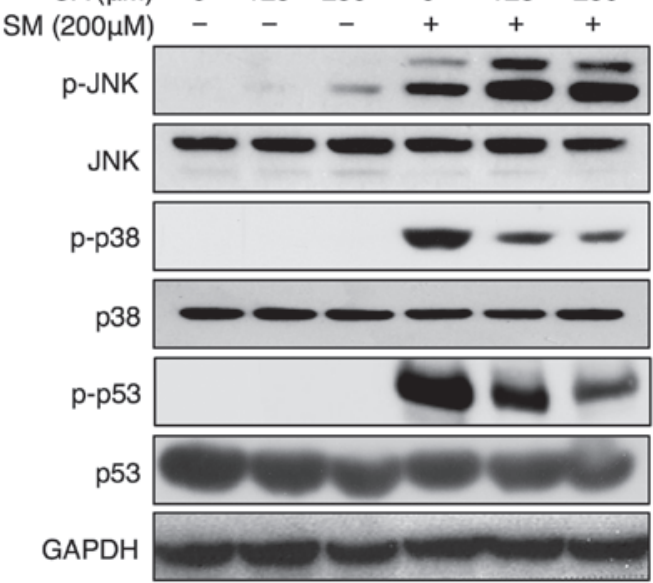

Figure 1. CA treatment reduces SM-induced cell death. NHEKs were treated with SM for 24 h. Cell viability assays were performed with (A) various SM concentrations ( $\mathrm{n}=4)$ and with (B) $200 \mu \mathrm{M}$ SM and the respective antioxidants $(\mathrm{n}=4)$. (C) Various concentrations of CA were co-incubated with SM, and a cell viability assay was performed ( $\mathrm{n}=4)$. (D) Western blots of NHEK lysates following $8 \mathrm{~h}$ treatment with SM with or without CA. The values are expressed as the mean \pm standard error of the mean. ${ }^{* *} \mathrm{P}<0.01 ;{ }^{* * *} \mathrm{P}<0.001$. Four independent experiments were performed. NHEKs, normal human epidermal keratinocytes; SM, sulfur mustard; TA, tannic acid; DFM, deferoxamine methylate; EA, elagic acid; CA, caffeic acid; NAC, N-acetyl cysteine; JNK, c-Jun N-terminal kinase; GAPDH, glyceraldehyde-3-phosphate dehydrogenase; p-, phosphorylated; O.D., optical density.

(250 $\mu \mathrm{M} \mathrm{CA}, 100 \mu \mathrm{M}$ quercetin or $250 \mu \mathrm{M}$ morin hydrate) for $8 \mathrm{~h}$, following which the NHEKs were washed with phosphate-buffered saline (PBS) twice and incubated with $5 \mu \mathrm{M}$ of DCF-DA for $30 \mathrm{~min}$ at $37^{\circ} \mathrm{C}$ in the dark. Following incubation, the NHEKs were washed with PBS twice and fluorescence intensity was detected with an excitation wavelength of $492 \mathrm{~nm}$ and emission of $530 \mathrm{~nm}$. Total nitrate/nitrite levels in the cell culture medium were quantified using a Nitrate/Nitrite Colorimetric Assay kit (Cayman Chemical Co., Ann Arbor, MI, USA) according to the manufacturer's protocol.

Statistical analysis. All experiments were repeated at least three times independently, and values are presented as the mean \pm standard error of the mean. Statistical significance was calculated using Student's t-test using Prism 6.0 software (GraphPad, San Diego, CA, USA). P<0.05 was considered to indicate a statistically significant difference.

\section{Results}

CA alleviates SM-induced cytotoxicity. To confirm the toxicity of SM in NHEK cells, various SM concentrations were used to treat NHEKs for $24 \mathrm{~h}$. As expected, SM treatment reduced cell viability in a dose-dependent manner, and cell viability was $\sim 50 \%$ at the $200 \mu \mathrm{M}$ concentration of SM (Fig. 1A). To identify potential therapeutic candidates for SM-induced cytotoxicity, several antioxidants were incubated with SM. Among these, CA $(250 \mu \mathrm{M})$ showed a protective effect against SM-induced cytotoxicity (Fig. 1B). In addition, CA treatment at concentrations $>250 \mu \mathrm{M}$ recovered cell viability in a dose-dependent manner (Fig. 1C). As SM has previously been reported to increase the phosphorylation of p38 MAP kinase and p53 $(1,18)$, the present study examined the MAP kinase and p53 pathways following treatment with SM and CA. SM treatment increased the phosphorylation of JNK1/2, p38 and $\mathrm{p} 53$, as reported previously $(1,18)$. CA treatment decreased the SM-induced phosphorylation of p38 and p53, and increased the phosphorylation of JNK 1/2 (Fig. 1D).

$C A$ reduces $S M$-induced cytotoxicity via the inhibition of $L O X$. To determine whether the p38 pathway is involved in the protective effect of CA on SM-induced cytotoxicity, a p38 inhibitor (SM203580) was co-incubated with SM and CA. p38 inhibition by SM203580 did not affect either SM-induced 
A

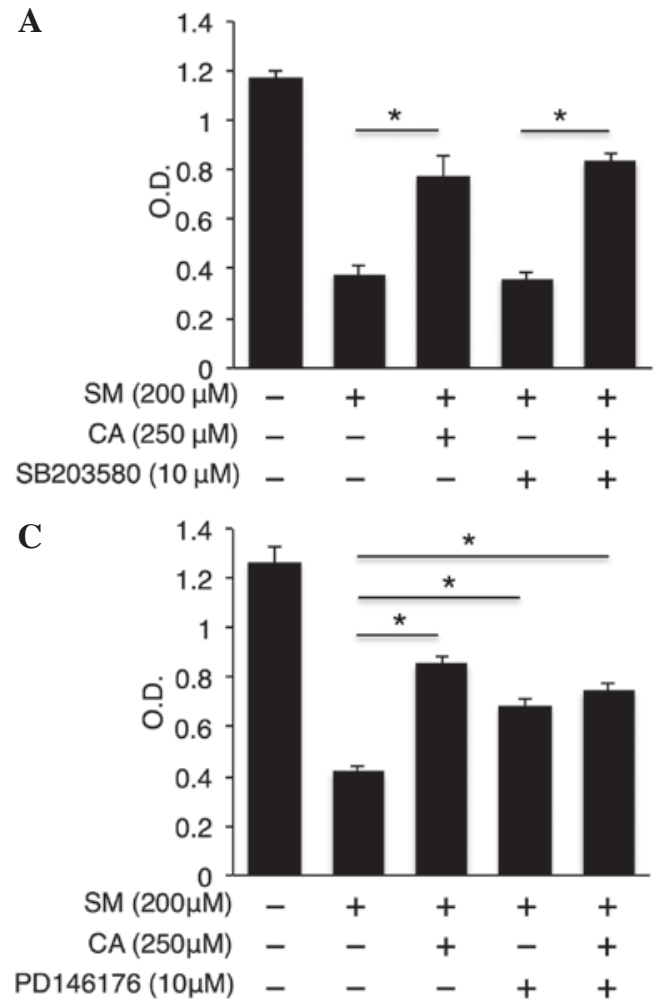

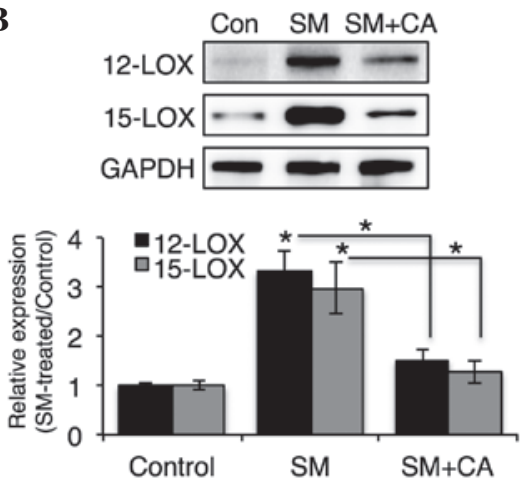

D

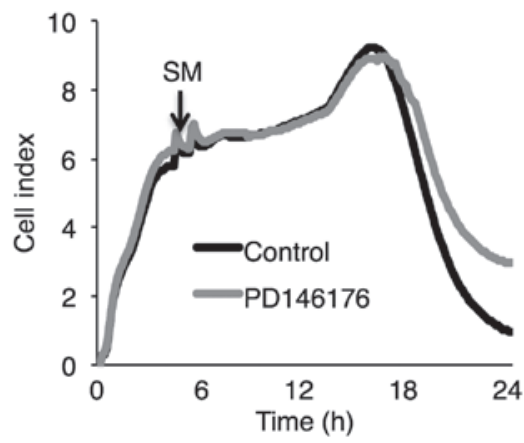

Figure 2. CA alleviates SM-induced cell death via the inhibition of 15-LOX. (A) Cell viability assay following co-treatment with $200 \mu$ M SM and CA or the p38 mitogen-activated protein kinase inhibitor, SB203580 (n=4). (B) Representative western blots of normal human epidermal keratinocyte lysates following co-treatment with SM $(200 \mu \mathrm{M})$ and CA, and their quantification $(\mathrm{n}=4)$. (C) Cell viability assay following co-treatment with $200 \mu \mathrm{M}$ SM and CA or the 15-LOX inhibitor, PD146176 (n=4). (D) Real-time cell electronic sensing assay following co-treatment with $200 \mu \mathrm{M}$ SM with or without PD146176. These experiments were repeated at least four times with similar results. Data are expressed as the mean \pm standard error of the mean, ${ }^{*}<0.05$. Four independent experiments are shown. CA, caffeic acid; SM, sulfur mustard; LOX, lipoxygenase; GAPDH, glyceraldehyde-3-phosphate dehydrogenase; Con control; O.D., optical density.

cytotoxicity or CA-induced cell recovery (Fig. 2A). As CA is also known as a LOX inhibitor (14), the present study examined whether the expression levels of LOX are altered upon SM treatment. Treatment with SM elevated the protein expression levels of 12- and 15-LOX, which were abrogated by CA treatment (Fig. 2B). In addition, treatment with the specific 15-LOX inhibitor, PD146176, significantly increased SM-treated cell viability, similar to the effects of CA (Fig. 2C), which confirmed the role of 15-LOX in SM-induced cytotoxicity. The RT-CES technique was used to confirm the improved cell viability by PD146176, the results of which also showed the protective effect of the 15-LOX inhibitor (PD146176) against SM (Fig. 2D).

Other plant-derived LOX inhibitors, morin hydrate and quercetin also reduce $S M$-induced cytotoxicity. To confirm whether the LOX pathway is involved in SM-induced cytotoxicity, other naturally obtained 15 -LOX inhibitors, including morin hydrate and quercetin (19), were co-incubated with SM. Treatment of cells with morin hydrate and quercetin partially recovered SM-induced cell death (Fig. 3A). Similarly, the RT-CES data showed delayed cell death upon morin hydrate and quercetin treatment (Fig. 3B). The reductions in the protein levels of 12- and 15 -LOX by incubation with morin hydrate and quercetin were confirmed using western blot analysis (Fig. 3C). Furthermore, morin hydrate and quercetin reduced the phosphorylation of $\mathrm{p} 38$ MAP kinase (Fig. 3C). However, PD146176 and SB203580 did not affect the expression levels of 12-LOX or 15-LOX, and only SB203580 reduced SM-induced p38 phosphorylation (Fig. 3D).

LOX inhibition reduces SM-induced levels of TNF- $\alpha$, but not $I L-1 \beta$. SM is also reported to elevate inflammatory cytokines, including TNF- $\alpha$ and IL-1 $\beta$ (6), thus, the present study measured the levels of TNF- $\alpha$ and IL-1 $\beta$ upon SM treatment with LOX inhibitors (CA, quercetin, morin hydrate and PD146176) or the p38 inhibitor (SB203580). p38 inhibition by SB203580 reduced the levels of TNF- $\alpha$ and IL-1 $\beta$ produced by SM, whereas the LOX inhibitors exerted different effects on cytokine production depending on their properties on the p38 pathway (Fig. 4A and B). Although all four LOX inhibitors reduced TNF- $\alpha$ levels, only $\mathrm{CA}$, quercetin and morin hydrate, which inhibited p38 MAP kinase (Fig. 3C), reduced the levels of IL-1 $\beta$ produced by SM. PD146176, which did not reduce SM-induced p38 phosphorylation (Fig. 3D), did not reduce the levels of SM-induced IL-1 $\beta$. All four LOX inhibitors decreased the SM-induced production of 15-HETE, as expected, which indicated effective LOX inhibition (Fig. 4C).

$C A$, quercetin and morin hydrate reduce the expression levels of COX2 and $i N O S$. Finally, the present study examined COX2 and iNOS signaling in SM-treated NHEKs. CA, quercetin and morin hydrate reduced the expression levels of COX2 and iNOS (Fig. 5A), whereas SB203580 and PD146176 did not affect the levels of COX2 or iNOS (Fig. 5B). In addition, 
A

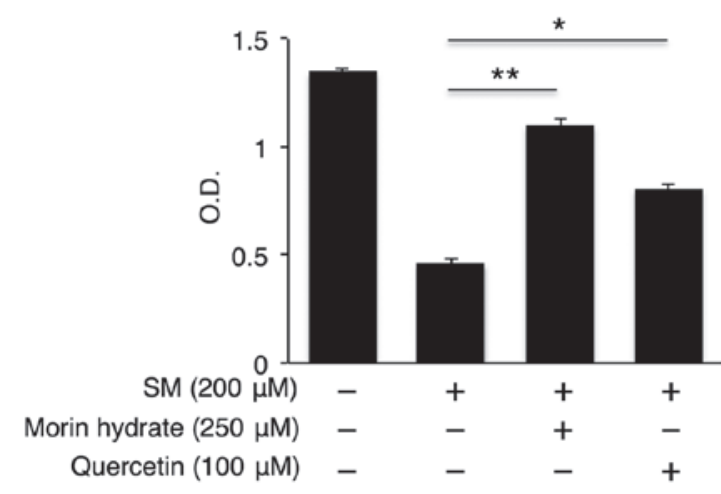

B
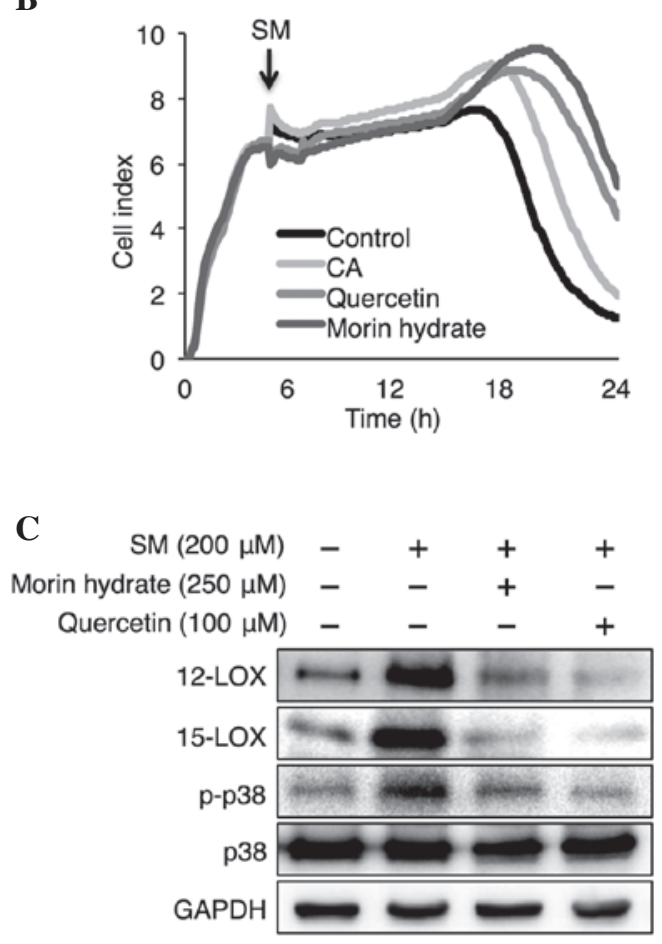

D

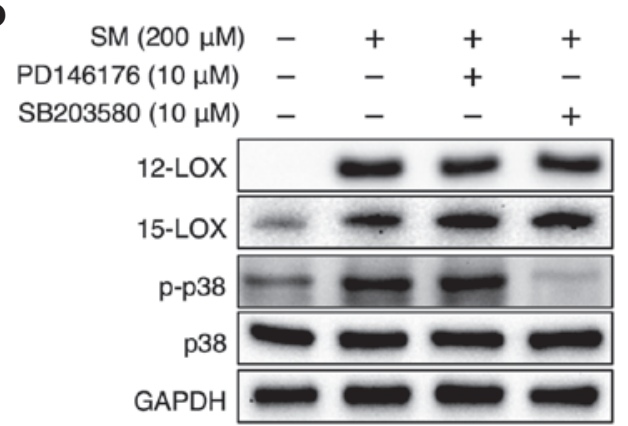

Figure 3. Natural 15-LOX inhibitors, quercetin and morin hydrate, have protective effects against SM-induced toxicity. (A) Cell viability assay following co-reatment with SM $(200 \mu \mathrm{M})$ and quercetin or morin hydrate $(n=4)$. (B) Real-time cell electronic sensing data of co-treatment with SM $(200 \mu \mathrm{M})$ and CA, quercetin or morin hydrate. Experiments were repeated at least four times with similar results. (C) Representative western blots of NHEK lysates following co-treatment with SM $(200 \mu \mathrm{M})$ and quercetin or morin hydrate, and (D) following co-treatment with SM $(200 \mu \mathrm{M})$ with PD146176 or SB203580. Data are expressed as the mean \pm standard error of the mean. ${ }^{*} \mathrm{P}<0.05$ and ${ }^{* *} \mathrm{P}<0.01$. Four independent experiments are shown. NHEKs, normal human epidermal keratinocytes; CA, caffeic acid; SM, sulfur mustard; LOX, lipoxygenase; GAPDH, glyceraldehyde-3-phosphate dehydrogenase; Con control; O.D., optical density; p-, phosphorylated.

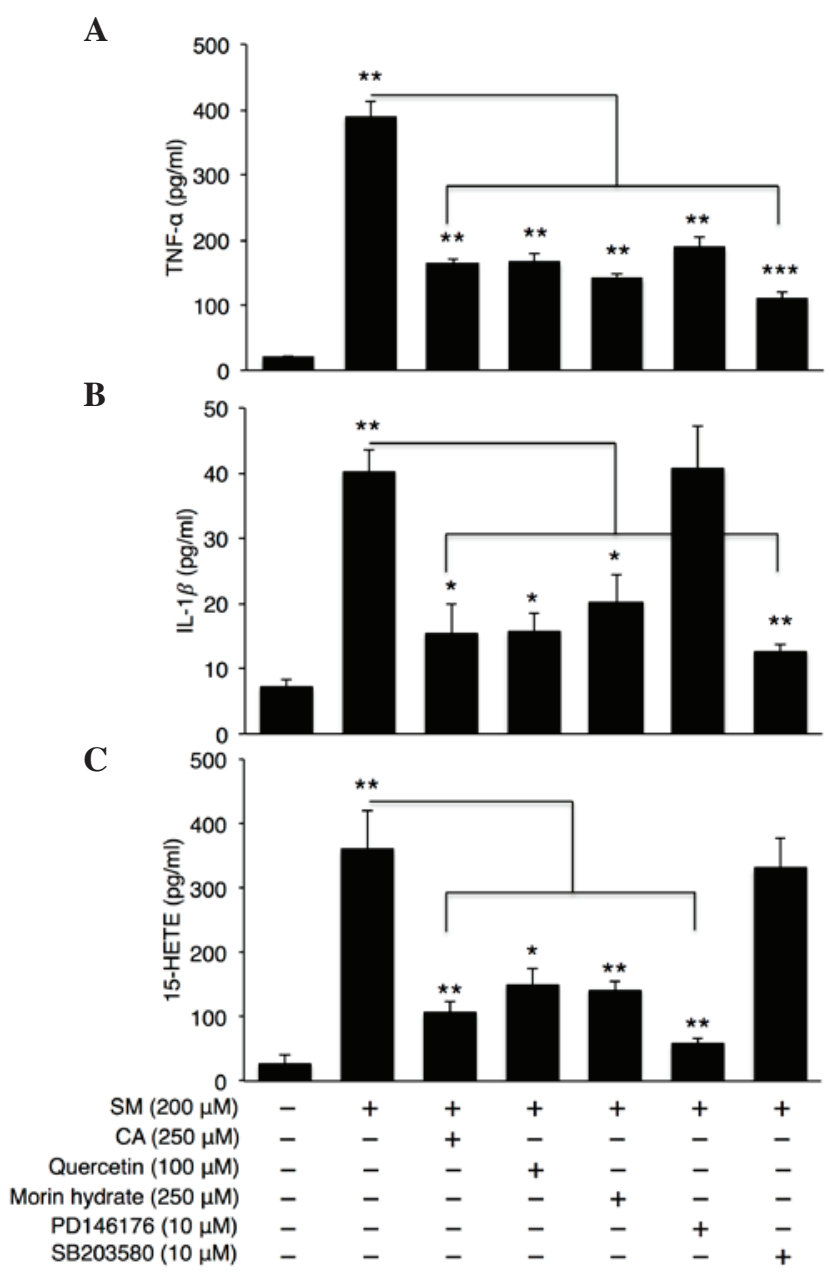

Figure 4. 15-LOX inhibition reduces levels of TNF- $\alpha$ and 15-HETE inflammatory cytokines. Normal human epidermal keratinocytes were treated with SM $(200 \mu \mathrm{M})$, antioxidants (CA, quercetin or morin hydrate) and specific inhibitors of p38 (SB203580) and 15-LOX pathways (PD146176) for $24 \mathrm{~h}$. Expression levels of (A) TNF- $\alpha$, (B) IL-1 $\beta$ and (C) 15-HETE were measured $(n=4)$. Data are expressed as the mean \pm standard error of the mean ${ }^{*} \mathrm{P}<0.05$, ${ }^{* *} \mathrm{P}<0.01$ and ${ }^{* * *} \mathrm{P}<0.001$. Four independent experiments are shown. LOX, lipoxygenase; SM, sulfur mustard; CA, caffeic acid; TNF- $\alpha$, tumor necrosis factor- $\alpha$; IL-1 $\beta$, interleukin - $1 \beta, 15$-HETE, hydroxyeicosatetraenoic acid.

CA, quercetin and morin hydrate attenuated nitrate/nitrite and ROS generation (Fig. 5C and D). From these results, it was concluded that $\mathrm{CA}$, quercetin and morin hydrate reduced COX2, iNOS and the generation of oxidative stress irrespective of the $\mathrm{p} 38$ and LOX pathway.

\section{Discussion}

Several mechanisms, including inflammation and oxidative stress, have been reported to be involved in SM-induced cytotoxicity $(6,8,20-22)$. For example, SM induces the production of inflammatory cytokines via p38 MAP kinase (6). SM also accelerates the release of arachidonic acids (21), which can be converted to prostaglandins and leukotrienes (23). SM exposure can also activate several signaling pathways, including the NF- $\mathrm{KB}$ and $\mathrm{p} 53$ pathway $(1,22)$. In the present study, it was found that the administration of CA, which has a LOX inhibitory effect, reduced the inflammatory response and cytotoxicity induced by SM. CA treatment decreased the phosphorylation 
A

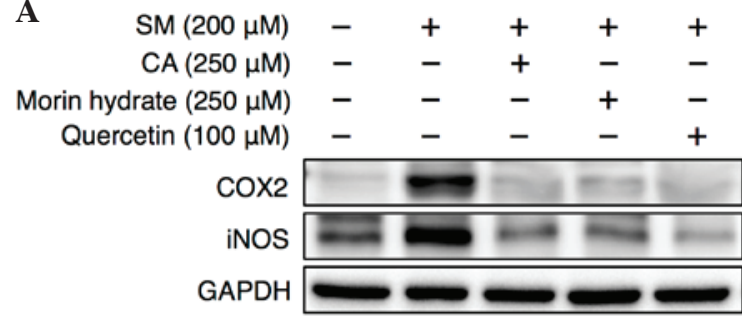

B

$\begin{array}{rllll}\mathrm{SM}(200 \mu \mathrm{M}) & - & + & + & + \\ \mathrm{PD} 146176(10 \mu \mathrm{M}) & - & - & + & - \\ \mathrm{SB} 203580(10 \mu \mathrm{M}) & - & - & - & + \\ \mathrm{COX} 2 & & \\ \mathrm{INOS} & & & \\ \text { GAPDH } & & & & \end{array}$

C

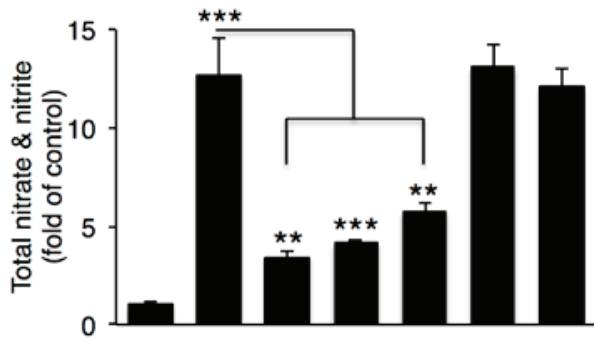

D

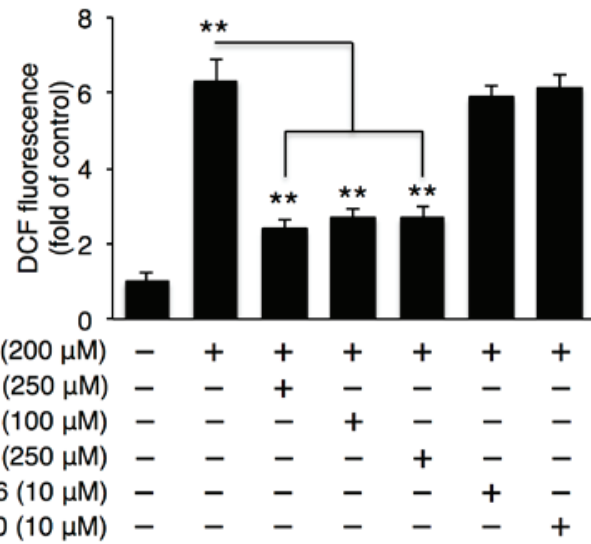

Figure 5. CA, quercetin and morin hydrate reduce ROS and nitrate/nitrite generation by SM. Representative western blots of normal human epidermal keratinocyte lysates following treatment with SM $(200 \mu \mathrm{M})$ and (A) CA, quercetin or morin hydrate, or (B) SB203580 or PD146176. (C) Total nitrate/nitrite and (D) ROS generation were measured following co-treatment with SM $(200 \mu \mathrm{M})$ and CA or quercetin or morin hydrate antioxidants $(\mathrm{n}=4)$. Data are expressed as the mean \pm standard error of the mean. ${ }^{* *} \mathrm{P}<0.01$ and ${ }^{* * *} \mathrm{P}<0.001$. Four independent experiments are shown. CA, caffeic acid; SM, sulfur mustard; LOX, lipoxygenase; ROS, reactive oxygen species; COX2, cyclooxygenase 2; GAPDH, glyceraldehyde-3-phosphate dehydrogenase; iNOS, inducible nitric oxide synthase; DCF, 2',7'-dichlorofluorescein.

of p38 MAP kinase and the elevation of LOX, resulting in decreased production of inflammatory cytokines and cytotoxicity. Furthermore, all LOX inhibitors, including quercetin, morin hydrate and PD146176, partially protected the NHEKs from SM-induced cell death. Several previous studies have reported the involvement of LOX in cell death (24-27). In fibroblasts, 12-LOX activation leads to apoptosis, and the 12-LOX product, 12-hydroperoxyeicosatetraenoic acid, directly induces apoptosis in CHO-AT1a cells (25). In addition, 12-LOX activation is important in oxidative stress-induced oligodendrocyte

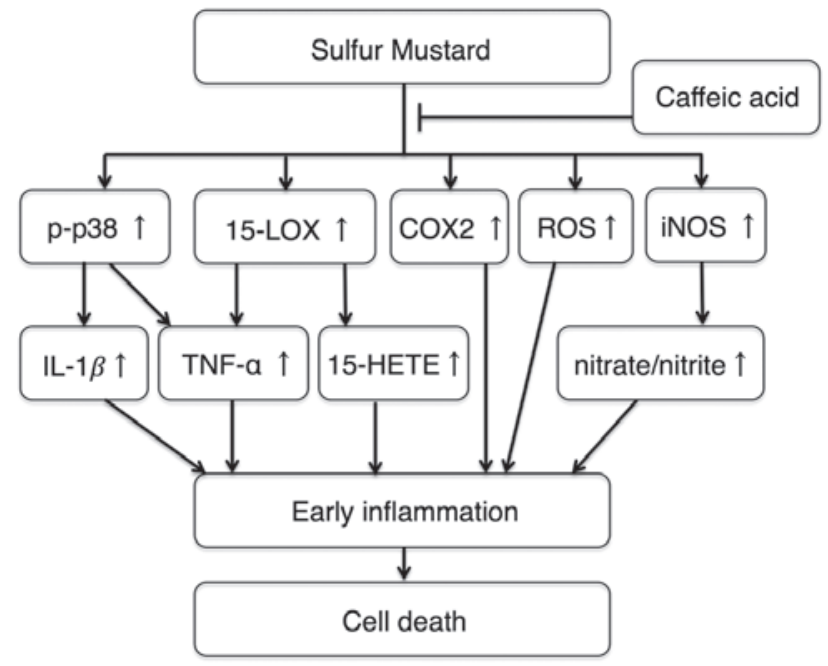

Figure 6. Schematic showing the effects of CA in sulfur mustard-induced toxicity. CA treatment reduces p38 phosphorylation, which reduces the levels of TNF- $\alpha$ and IL-1 $\beta$. 15-LOX inhibition by CA attenuates the production of TNF- $\alpha$ and 15-HETE. CA also reduces the levels of COX2 and iNOS, and decreases ROS and nitrate/nitrite generation. These effects decrease SM-induced inflammation and cell death. CA, caffeic acid; LOX, lipoxygenase; COX2, cyclooxygenase 2; ROS, reactive oxygen species; iNOS, inducible nitric oxide synthase; IL-1 $\beta$, interleukin $-1 \beta, \mathrm{TNF}-\alpha$, tumor necrosis factor- $\alpha$; 15-HETE, hydroxyeicosatetraenoic acid.

death (26), and the activation of neuronal 12-LOX leads to the production of peroxides and influx of $\mathrm{Ca}^{2+}$, ultimately leading to cell death (27). Additionally, 12/15-LOX-derived lipid peroxidation triggers apoptosis-inducing factor-mediated cell death (24). The results of the present study suggested that the LOX pathway was also involved in SM-induced cell death, and that inhibiting the LOX pathway offers a potential therapeutic target for SM-induced toxicity.

A crucial role of the 5-LOX/12-LOX/15-LOX pathway in regulating the production of TNF- $\alpha$ and TNF- $\alpha$-mediated inflammation has been previously reported (28-30). In accordance with these previous studies, the present study showed that $\mathrm{CA}$, quercetin and morin hydrate decreased the production of TNF- $\alpha$, not only by the p38 pathway, but also through the inhibition of 15-LOX. However, unlike the production of TNF- $\alpha$, inhibition of the p38 pathway, but not of 15-LOX, reduced the production of IL-1 $\beta$. Furthermore, CA, quercetin and morin hydrate reduced the generation of ROS and nitrate/nitrite. As neither p38 inhibition nor 15-LOX inhibition affected the generation of ROS or nitrate/nitrite, these effects may be derived from their scavenging effects of free radicals (31-33).

Although the activation of MAP kinases, including p38 and JNK-1/-2, was induced by SM, CA treatment reduced the phosphorylation of p38 MAP kinase, but not JNK-1/-2. In a previous study, CA was reported to paradoxically increase the phosphorylation of JNK (34). As CA treatment did not reduce SM-induced JNK phosphorylation, the precise role of JNK activation in SM-induced cytotoxicity remains to be elucidated.

Various mechanisms involved in SM-induced toxicity have been reported (1) and current knowledge of the mechanism underlying SM-induced toxicity is increasing. Although the development of therapeutic agents against SM is complex due 
to the complexity of the mechanisms involved, a combinational approach may lead to an effective treatment strategy for SM-induced toxicity. The present study suggested that natural LOX inhibitors, including CA, quercetin and morin hydrate, can be used as early therapeutic agents for SM-induced toxicity by reducing the early inflammatory responses, oxidative stress and expression of COX2/iNOS induced by SM (Fig. 6).

\section{Acknowledgements}

All experiments were performed between 2009 and 2011. Joo-Won Park was supported by the Health Technology R\&D project (grant no. HI14C2445) of the Ministry of Health and Welfare, Republic of Korea. Woo-Jae Park was supported by a Gachon University Research Grant of 2015 (grant no. GCU-2015-5112). Shin Kim was supported by the National Research Foundation of Korea Grant funded by the Korean Government (grant. no. 2014R1A5A2010008)

\section{References}

1. Kehe K, Balszuweit F, Steinritz D and Thiermann H: Molecular toxicology of sulfur mustard-induced cutaneous inflammation and blistering. Toxicology 263: 12-19, 2009.

2. Ries C, Popp T, Egea V, Kehe K and Jochum M: Matrix metalloproteinase-9 expression and release from skin fibroblasts interacting with keratinocytes: Upregulation in response to sulphur mustard. Toxicology 263: 26-31, 2009.

3. McClintock SD, Hoesel LM, Das SK, Till GO, Neff T, Kunkel RG, Smith MG and Ward PA: Attenuation of half sulfur mustard gas-induced acute lung injury in rats. J Appl Toxicol 26: 126-131, 2006

4. Kehe K, Raithel K, Kreppel H, Jochum M, Worek F and Thiermann H: Inhibition of poly (ADP-ribose) polymerase (PARP) influences the mode of sulfur mustard (SM)-induced cel death in HaCaT cells. Arch Toxicol 82: 461-470, 2008.

5. Simbulan-Rosenthal CM, Ray R, Benton B, Soeda E, Daher A, Anderson D, Smith WJ and Rosenthal DS: Calmodulin mediates sulfur mustard toxicity in human keratinocytes. Toxicology 227: 21-35, 2006.

6. Dillman JF III, McGary KL and Schlager JJ: An inhibitor of p38 MAP kinase downregulates cytokine release induced by sulfur mustard exposure in human epidermal keratinocytes. Toxicol In Vitro 18: 593-599, 2004

7. Rebholz B, Kehe K, Ruzicka T and Rupec RA: Role of NF-kappaB/RelA and MAPK pathways in keratinocytes in response to sulfur mustard. J Invest Dermatol 128: 1626-1632, 2008.

8. Pal A, Tewari-Singh N, Gu M, Agarwal C, Huang J, Day BJ, White CW and Agarwal R: Sulfur mustard analog induces oxidative stress and activates signaling cascades in the skin of SKH-1 hairless mice. Free Radic Biol Med 47: 1640-1651, 2009.

9. Paromov V, Suntres Z, Smith M and Stone WL: Sulfur mustard toxicity following dermal exposure: Role of oxidative stress and antioxidant therapy. J Burns Wounds 7: e7, 2007.

10. Kumar O, Sugendran K and Vijayaraghavan R: Protective effect of various antioxidants on the toxicity of sulphur mustard administered to mice by inhalation or percutaneous routes. Chem Biol Interact 134: 1-12, 2001

11. Naghii MR: Sulfur mustard intoxication, oxidative stress, and antioxidants. Mil Med 167: 573-575, 2002.

12. Gamaro GD, Suyenaga E, Borsoi M, Lermen J, Pereira P and Ardenghi P: Effect of rosmarinic and caffeic acids on inflammatory and nociception process in rats. ISRN Pharmacol 2011: $451682,2011$.

13. Natarajan K, Singh S, Burke TR Jr, Grunberger D and Aggarwal BB: Caffeic acid phenethyl ester is a potent and specific inhibitor of activation of nuclear transcription factor NF-kappa B. Proc Natl Acad Sci USA 93: 9090-9095, 1996.

14. Sud'ina GF, Mirzoeva OK, Pushkareva MA, Korshunova GA, Sumbatyan NV and Varfolomeev SD: Caffeic acid phenethyl ester as a lipoxygenase inhibitor with antioxidant properties. FEBS Lett 329: 21-24, 1993.
15. Sultana B and Anwar F: Flavonols (kaempeferol, quercetin, myricetin) contents of selected fruits, vegetables and medicinal plants. Food Chem 108: 879-884, 2008.

16. Rattanachaikunsopon P and Phumkhachorn P: Bacteriostatic effect of flavonoids isolated from leaves of Psidium guajava on fish pathogens. Fitoterapia 78: 434-436, 2007.

17. Brash AR: Lipoxygenases: Occurrence, functions, catalysis, and acquisition of substrate. J Biol Chem 274: 23679-23682, 1999.

18. Minsavage GD and Dillman JF III: Bifunctional alkylating agent-induced p53 and nonclassical nuclear factor kappaB responses and cell death are altered by caffeic acid phenethyl ester: A potential role for antioxidant/electrophilic response-element signaling. J Pharmacol Exp Ther 321: 202-212, 2007.

19. Sadik CD, Sies H and Schewe T: Inhibition of 15-lipoxygenases by flavonoids: Structure-activity relations and mode of action. Biochem Pharmacol 65: 773-781, 2003.

20. Black AT, Joseph LB, Casillas RP, Heck DE, Gerecke DR, Sinko PJ, Laskin DL and Laskin JD: Role of MAP kinases in regulating expression of antioxidants and inflammatory mediators in mouse keratinocytes following exposure to the half mustard, 2-chloroethyl ethyl sulfide. Toxicol Appl Pharmacol 245: 352-360, 2010.

21. Lefkowitz LJ and Smith WJ: Sulfur mustard-induced arachidonic acid release is mediated by phospholipase D in human keratinocytes. Biochem Biophys Res Commun 295: 1062-1067, 2002.

22. Gerecke DR, Chen M, Isukapalli SS, Gordon MK, Chang YC, Tong W, Androulakis IP and Georgopoulos PG: Differential gene expression profiling of mouse skin after sulfur mustard exposure: Extended time response and inhibitor effect. Toxicol Appl Pharmacol 234: 156-165, 2009.

23. Folco G and Murphy RC: Eicosanoid transcellular biosynthesis: From cell-cell interactions to in vivo tissue responses. Pharmacol Rev 58: 375-388, 2006.

24. Seiler A, Schneider M, Förster H, Roth S, Wirth EK, Culmsee C, Plesnila N, Kremmer E, Rådmark O, Wurst W, et al: Glutathione peroxidase 4 senses and translates oxidative stress into 12/15-lipoxygenase dependent- and AIF-mediated cell death. Cell Metab 8: 237-248, 2008.

25. Gu J, Liu Y, Wen Y, Natarajan R, Lanting L and Nadler JL: Evidence that increased 12-lipoxygenase activity induces apoptosis in fibroblasts. J Cell Physiol 186: 357-365, 2001.

26. Wang H, Li J, Follett PL, Zhang Y, Cotanche DA, Jensen FE, Volpe JJ and Rosenberg PA: 12-Lipoxygenase plays a key role in cell death caused by glutathione depletion and arachidonic acid in rat oligodendrocytes. Eur J Neurosci 20: 2049-2058, 2004.

27. Li Y, Maher P and Schubert D: A role for 12-lipoxygenase in nerve cell death caused by glutathione depletion. Neuron 19: 453-463, 1997.

28. Martínez-Clemente M, Ferré N, González-Périz A, López-Parra M, Horrillo R, Titos E, Morán-Salvador E, Miquel R, Arroyo V, Funk CD and Clària J: 5-lipoxygenase deficiency reduces hepatic inflammation and tumor necrosis factor alpha-induced hepatocyte damage in hyperlipidemia-prone ApoE-null mice. Hepatology 51: 817-827, 2010.

29. Wen Y, Gu J, Chakrabarti SK, Aylor K, Marshall J, Takahashi Y, Yoshimoto T and Nadler JL: The role of 12/15-lipoxygenase in the expression of interleukin- 6 and tumor necrosis factor-alpha in macrophages. Endocrinology 148: 1313-1322, 2007.

30. Schade UF, Ernst M, Reinke M and Wolter DT: Lipoxygenase inhibitors suppress formation of tumor necrosis factor in vitro and in vivo. Biochem Biophys Res Commun 159: 748-754, 1989.

31. Zhang R, Kang KA, Piao MJ, Maeng YH, Lee KH, Chang WY, You HJ, Kim JS, Kang SS and Hyun JW: Cellular protection of morin against the oxidative stress induced by hydrogen peroxide. Chem Biol Interact 177: 21-27, 2009.

32. Dugas AJ, Castañeda- Acosta J, Bonin GC, Price KL, Fischer NH and Winston GW: Evaluation of the total peroxyl radical-scavenging capacity of flavonoids: Structure-activity relationships. J Nat Prod 63: 327-331, 2000.

33. Chen YJ, Shiao MS and Wang SY: The antioxidant caffeic acid phenethyl ester induces apoptosis associated with selective scavenging of hydrogen peroxide in human leukemic HL-60 cells. Anticancer Drugs 12: 143-149, 2001.

34. Choi K and Choi C: Differential regulation of c-Jun N-terminal kinase and NF-kappaB pathway by caffeic acid phenethyl ester in astroglial and monocytic cells. J Neurochem 105: 557-564, 2008. 